\title{
Research on the Automotive Remote Failure Diagnosis based on Complex Network Theory
}

\author{
Haibin Zhao ${ }^{\text {, }}$ Aibing Wang \\ Hebei Jiaotong Vocational \& Technical College, Shijiazhuang 050091, China; \\ apaperiset@163.com
}

Keywords: automotive, remote failure diagnosis, complex networks.

\begin{abstract}
First, this paper analyzes the research status of vehicle networking, engineering applications, electronic control engine fault diagnosis and develops research content and design route for this paper. From view of application, this paper analyzes the complex network model, complex network rules, complex network structure designs, and the selection rules for initial value as well as the diagnostic process. The paper also presents the disadvantages of applying complex networks to failure diagnosis and its corrective methods..
\end{abstract}

\section{Introduction}

With the rapid development of economic and modernization of social, the scale of automobile's production are increased widely, and the use of automobile are also more widely. Use fault diagnosis system can help automotive repair business to diagnose the cause of the malfunction quickly and efficiently when the car has faults, and repair the faults in time, so as to ensure the safety and reliability of the car. [1] The traditional a single model of fault diagnosis cannot meet the needs of made diagnosis clipping and accurate, because of the complexity structure of the car and many kinds of faults, [2] therefore, this paper gives an automotive fault diagnosis system based on complex network theory.

The extensive study on complex networks is pervading sciences and engineering today, from physical, technological, biological, to social sciences. [3] Their impacts on engineering and technology, in particular, are prominent and their influence is deemed to be far-facing. Familiar complex networks include the traffic network, wireless communication networks, biological neural networks, power grids, social relation and scientific cooperation networks and so on. Research on fundamental properties and dynamical behaviors of various complex networks has become overwhelming recently.

The development of the mathematical graph theory has a very slow start after Euler solved the Konigsburg seven-bridge problem. The first monograph on graph theory was published exactly two-hundred later, in 1936. Nevertheless, the theory was developed rather rapidly thereafter, and the foundation of the now famous "random graph theory" was laid by two Hungarian mathematicians, Paul Erdos (1913-1970), As a historical remark, Pual Erdos is one of the most distinguishes leading mathematicians of the twentieth century, in the late 1950s, which is considered the first rigorous and complete modern graph theory.

Since every possible pair of nodes is picked up once and once only, there will not be multiple edges between any pair of nodes and, moreover, no node has a self-connected edge.

It can be easily seen that in such an ER random graph of $N$ nodes, the expectation value of the total number of edges will be $N(N-1) p / 2$, which is a random variable because $p$ is so. Consequently, the probability of obtaining a graph with $N$ nodes and $M$ edges is equal to $p^{M}(1-p)^{N(N-1) / 2-M}$. Erdos and Renyi systematically studied many asymptotic properties, as $N \rightarrow \infty$, of such graphs and their relations with the edge-connectivity probability $p$. If a graph has a property $P$ with probability 1 as $N \rightarrow \infty$, then they consider almost every ER random graph has property $P$. One of theory most important and also quite surprising discoveries is that many properties of ER random graphs emerge suddenly but not gradually, in the sense that for a given edge-connectivity probability $p$, either 
almost every ER random graph has a certain property $P$ or almost every such graph does not have property $P$.

\section{Small world model}

Model assumptions. From a statistical point of view, although the network of automotive failure diagnose can be computed, they are nevertheless too small to be conclusive. Knowing this, trying to carry out a large-scale international experiment to verify the hypothesis of small world. We selected some targeted automotive, with different brand, 4S shop. Notice that the small world algorithm may destroy the network connectivity during the rewiring process, yielding possible some unconnected clusters. As a remedy, the small world algorithm is as follows:

(1)Start from a ring shaped network with $N$ nodes, in which each node is connected to its $2 K$ neighbors, where $K>0$ is an integer (usually small).

(2)For every pair of originally unconnected nodes, with probability $p$, add an edge to connect them.

Theorem 1. For large enough size $N$, the clustering coefficient of the small world network model is given by

$$
C(p)=\frac{3(K-1)}{2(2 K-1)}(1-p)^{3}
$$

For $p=0$, each node has $2 K$ neighbours, so the number of edges among these neighbours is $N_{0}=3 K(K-1) / 2$, while the number of all possible edges among these nodes is $\left.2 K(k-1) / 2\right)$; therefore, $C(0)=3(K-1) /(2(2 K-1))$. For $p>0$, two neighbours of node $i$, which were connected at $p=0$ are still neighbours of $i$ and remain being connected with probability $(1-p)^{3}$, up to some terms of order $O(1 / N)$. Thus, the mean number of edges among the neighbours of a node is $N_{0}=(1-p)^{3}+O(1 / N)$. Consequently, the clustering coefficient is given by $N_{0}(1-p)^{3} / K(2 K-1)$.

Theorem 2. The average path length of the small world network model is given by [3]

$$
L(p)=\frac{2 N}{K} f(2 N p / K)
$$

With

$$
f(x)=\left\{\begin{array}{cc}
c & x<1 \\
\ln x / x & x>1
\end{array}\right.
$$

The average path length of the small world network model is also given by [4]

$$
f(x) \approx \frac{1}{2 \sqrt{x^{2}+2 x}} \operatorname{artanh} \sqrt{\frac{x}{x^{2}+2 x}}
$$

For the network model, with $p$ fixed, first perform the renormalization process and let the number of sites of the resultant renormalized network be $\mathrm{S}$. the average path length $L(p)$ is less than 1 and is increasing linearly as $\mathrm{S}$ gradually increases. Bur at some threshold value of $\mathrm{S}^{*}, L(p)$ will become bigger than 1 . This leads to a phase transition, after which $L(p)$ will increase only logarithmically. To be more precise, consider only the case of $\mathrm{K}=1$, namely, a perfect ring, and assume that $0<p<1$ and $S^{*}=1 / p$, thus $S^{*}>1$. In this case, $L(p)$ should obey a finite-size scaling law of the form $L(p)=S f\left(S / S^{*}\right)=S f(p S)$. From the renormalized network, it can be seen that $S=2 N / K$.

\section{Experiment}

In the future, vehicles communicate with vehicles though wireless communication technology, $\mathrm{V} 2 \mathrm{~V}$ is a big network based on large data. In recent years, along with the rapid development of the Internet and the Internet of Things, vehicle networking has become a major signal, announcing that ears have entered into an intelligent era. The rapid development and popularization of vehicle networking technologies all over the world, has provided the conditions and foundation for remote fault diagnosis technology. 
Table 1 Examples of road running status at the moment

\begin{tabular}{clll}
\hline Numble & DAYTAG & MINTAG & COUNT \\
\hline 1 & $2015-10-01$ & 0 & 2 \\
2 & $2015-10-01$ & 1 & 1 \\
3 & $2015-10-01$ & 2 & 1 \\
\hline
\end{tabular}

The engine is the power source of the vehicle with poor operating conditions, which results in relatively high failure rate. The structure of electronic control engine is more complex with more complicated failures and more difficulties of fault diagnosis. However, so far, knowledge for automotive electronic control engine fault diagnosis is often heterogeneous and lacking semantic association between each other. Therefore, there is no universal conceptual model that can be commonly understood, which results in difficulty to knowledge acquisition, expression, sharing and reuse. Experimental procedure and data obtained are shown in Table 1 and Table 2.

\begin{tabular}{clll}
\multicolumn{4}{c}{ Table 2 Examples of failure diagnose status at the moment } \\
\hline Numble & DAYTAG & MINTAG & COUNT \\
\hline 1 & $2015-10-01$ & 0 & 14 \\
2 & $2015-10-01$ & 1 & 1 \\
3 & $2015-10-01$ & 2 & 10 \\
4 & $2015-10-01$ & 3 & 2 \\
5 & $2015-10-01$ & 4 & 12 \\
6 & $2015-10-01$ & 5 & 1 \\
7 & $2015-10-01$ & 6 & 15 \\
8 & $2015-10-01$ & 7 & 5 \\
9 & $2015-10-01$ & 8 & 18 \\
10 & $2015-10-01$ & 9 & 3 \\
\hline
\end{tabular}

With MATLAB7.0.1 data-calculation software as the assistance, this paper mainly studies the failure diagnosis emulation of auto engines, which includes the access to the sample set, the design of the network, the pre-treatment of information. It also makes a study on the trouble shooting of the misfire of an engine with the technology of complex network and ELMAN network. A comparison between complex network and ELMAN network is made and studied, which reaches a conclusion that the error amount of complex network is smaller than that of ELMAN network. But due to the feedback effect of ELMAN network, its error curve is smooth and tends to be a straight line, complex network can respond automotive fault diagnostic work fully. This article also use the powerful data calculation functions of MATLAB and the powerful interactive features of VB, using Active $\} \mathrm{C}$ technology between VB and MATLAB to achieve the seamless integration, completing the development of fault diagnosis system, With the help of VB and SQL's integration to achieve the fault system database modifications and updates. Comparison table of colour-failure level index range and test group is shown in Table.

Table 3 Comparison table of color-failure level index range

\begin{tabular}{ccccccc}
\hline & & & & & & \\
Level & No data & Trouble free & Minor fault Moderate faults & Serious & Unable to travel \\
index & -- & {$[0,2]$} & $(2,4]$ & $(4,6]$ & $(6,8]$ & $(8,10]$ \\
\hline
\end{tabular}

We have tested our strategy with different influencing factors concering randomness of automotive failure diagnose network, the network model (Fig. 1) and clustering coefficient with failure rate (Fig. 2) 


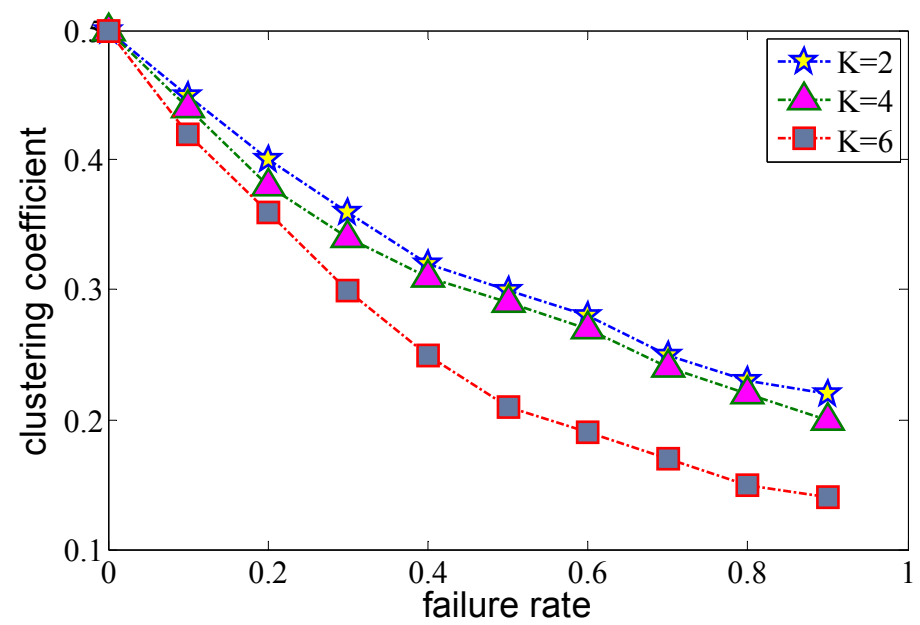

Fig. 1 The cluttering coefficient reduction results using our strategy, in basic network with node number $\mathrm{K}=2,4,6$ respectively, when generating the small world network. ( $\mathrm{S}=18, \mathrm{~N}=1000$ )

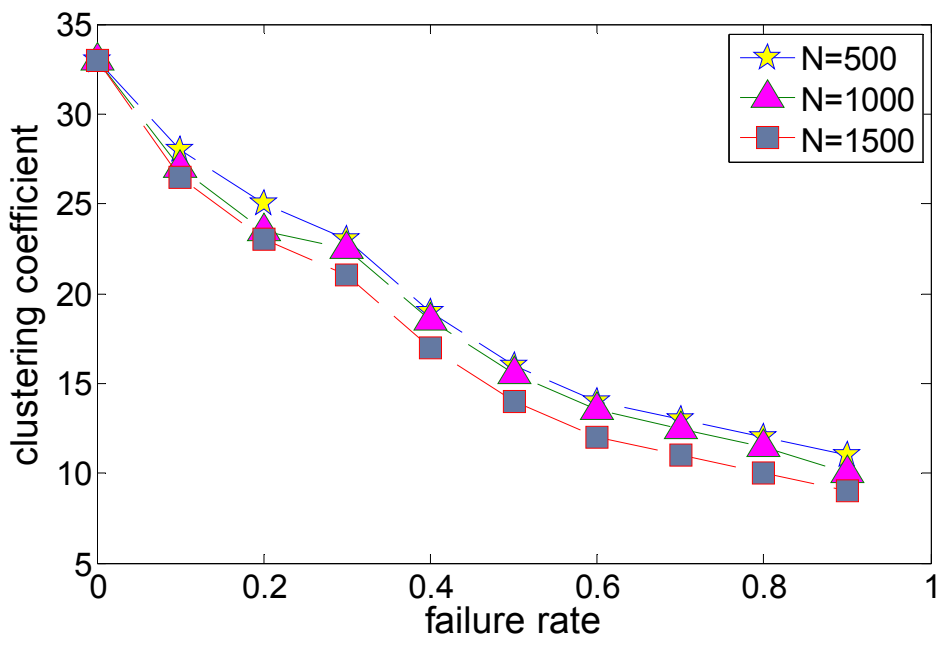

Fig. 2 The cluttering coefficient reduction results using our strategy, with $\mathrm{N}=500,1000,1500$ respectively, when generating the small world network, connecting node pairs randomly with one node added and our strategy making random connections instead. $(\mathrm{S}=18, \mathrm{~K}=2)$

Recall that in the small world network model, each node on the given ring shaped small world network has long range edges connecting to the other nodes with a certain probability in such a uniform random manner that every other node has an equal probability to receive the new edge, excluding self loops and multiple connections. Now, instead of assuming that new long range connections are uniformly distributes over the whole network, we argue that intuitively it would be easier for a new edge to connect to a nearly neighbour than to those remote ones, therefore assume that the connecting probability is reversely proportional to the distance between the two nodes. More specifically, we obtain the relationship between $N$ and average path.

\section{Summary}

In order to research the mechanism of automotive failure diagnose and improve the effective, as well as to explore a new perspective to a find automotive failure diagnose quickly, this paper based on the empirical data to analyze Wuhan's some 4S shop and its self-organized criticality proposed a new suggestion. In this paper, we analyze in depth the data of automotive failure running status and diagnose index of different period between 2014, based on the theory of automotive failure diagnose complexity and self-organized criticality, and thus prove the characteristic of power-law under which lies the related scale. The result shows us that, automotive failure diagnose system is a dynamical system that's both extensive and dissipative. In addition, when STATUS is under 20 or less and TPI is above 6 , the scale of influenced districts caused by index in automotive diagnose system and the 
related frequency fits the law-power distribution, and the rising of automotive will reach the state of self-organized criticality, and meets the characteristic of self-organized criticality

\section{References}

[1] JIANG Ke-jin, ZHANG Dian-ye. The Study of Urban Road Traffic System's Self-Organization Model and Simulation [J]. Ergonomics, 2008, 14(2): 37-40.

[2] WANG Bing-hong, MAO Dan, WANG Lei. Research on Self-Organized Criticality Study of Traffic Flow [J]. The Academic Journal of Guangxi Normal University ( Natural Science Edition).

[3] Bak P, Tang C Wiesenfeld K. Self-organized criticality: an explanation of 1/f noise [J]. Physical Review Letters. 1987, 59: 381-384.

[4] YU Qun. The Study of Self-Organized Criticality in Power System's Blackouts of Our Country[J]. Smat-Grid Technology, 2006, 30(6): 1-5. 